Notfall Rettungsmed 2015 · 18:231

DOI 10.1007/s10049-015-0004-1

Online publiziert: 21. April 2015

c) Springer-Verlag Berlin Heidelberg 2015

\title{
Europäische Facharztprüfung für Notfallmedizin
}

\section{Zum Beitrag \\ Dißmann PD (2014) Europäische Facharztprü- \\ fung für Notfallmedizin. Erfahrungsbericht \\ und Empfehlungen zur Prüfungsvorberei- \\ tung. Notfall Rettungsmed 17:697-700}

\section{Leserbrief}

\section{Hohenstein}

Zentrale Notaufnahme, Universitätsklinikum Jena, Deutschland

Ich danke Dr. Dißmann herzlich für die Ausführungen über die neu initiierte Facharztprüfung Notfallmedizin durch die Europäische Gesellschaft für Notfallmedizin. Dies ist ein wichtiger Schritt, die Notfallmedizin nicht als die präklinische Rettungsmedizin und deren Anforderungen zu betrachten, sondern die Präklinik als kleinen Teil eines großen Fachgebiets Notfallmedizin. Dass wir in Deutschland allerdings noch einen sehr weiten Weg vor uns haben, kommt durch die von Dr. Dißmann angegebenen Prüfungsergebnisse nicht klar zum Ausdruck. Es haben 7 Deutsche die schriftliche Prüfung bestanden, und hiervon aber nur 3 die mündliche, obwohl „Brot-und-Butter-Fälle“ der Notaufnahme geprüft wurden. Allerdings hat keiner dieser 3 Kandidaten einen deutschen Facharzttitel. Die 3 Kollegen leben zwar in Deutschland, ihre Ausbildung absolvierten sie aber in Großbritannien und Australien in der Notaufnahme. Trotz des vom Autor durchgeführten Vorbereitungskurses sind alle deutschen Fachärzte, die ausnahmslos und jahrelang in wirklich interdisziplinär arbeitenden Notaufnahmen tätig sind, chancenlos dem angloamerikanischen Prüfungssystem „OSCE“ unterlegen gewesen, obwohl alle an diesem Vorbereitungskurs teilnahmen. Insofern liegen die Prüfungsergebnisse der Deutschen nicht über dem Durchschnitt, sondern mit einer Bestehensquote von $0 \%$ maximal unter dem Durchschnitt.
Ein einziger Prüfungsvorbereitungskurs wird diesen Missstand in der nahen $\mathrm{Zu}$ kunft kaum flächendeckend lösen können - und um die Flächendeckung geht es hier. Um diese Prüfung bestehen zu können, und breites notfallmedizinisches Wissen reicht ganz offensichtlich hierfür nicht aus, werden wir in Deutschland das gesamte Ausbildungssystem der Notfallmedizin auch im klinischen Alltag ändern müssen. Eine Änderung des Prüfungsstils ist nach Aussage des Autors nicht in Sicht. Ich kann nur hoffen, dass es viele Kolleg(inn)en geben wird, die sich hierauf einlassen und möchte hierzu ermuntern. Denn, wie richtig erwähnt, geht es nicht nur um die breite fachliche Qualifikation, sondern zusätzlich um den korrekten Umgang mit „OSCE's“. Eine Masse an europäischen Fachärzten für Notfallmedizin, sog. „FEBEM's" (Fellow of European Board of Emergency Medicine), ist für Deutschland sicher ein Vorteil.

Dr. med. Christian Hohenstein

\section{Korrespondenzadresse}

\section{Dr. C. Hohenstein}

Zentrale Notfallaufnahme

Universitätsklinikum Jena, Jena

christian.hohenstein@med.uni-jena.de 